\title{
Q.
QNEEN'S
UNIVERSITY
BELFAST
}

\section{DEM Modelling of a New 'Sphere Filling' Approach for Optimising Motion Control of Rotational Moulding Processes}

Adams, J., Jin, Y., Barnes, D., \& Butterfield, J. (2018). DEM Modelling of a New 'Sphere Filling' Approach for Optimising Motion Control of Rotational Moulding Processes. In S. Wang, M. Price, M. K. Lim, Y. Jin, Y. Luo, \& R. Chen (Eds.), Recent Advances in Intelligent Manufacturing: First International Conference on Intelligent Manufacturing and Internet of Things and 5th International Conference on Computing for Sustainable Energy and Environment, IMIOT and ICSEE 2018, Chongqing, China, September 21-23, 2018, Proceedings, Part I (Vol. 923, pp. 239-248). (Communications in Computer and Information Science ). Springer. https://www.springer.com/gb/book/9789811323959

Published in:

Recent Advances in Intelligent Manufacturing

Document Version:

Peer reviewed version

Queen's University Belfast - Research Portal:

Link to publication record in Queen's University Belfast Research Portal

Publisher rights

Copyright 2018 Springer. This work is made available online in accordance with the publisher's policies. Please refer to any applicable terms of use of the publisher.

\section{General rights}

Copyright for the publications made accessible via the Queen's University Belfast Research Portal is retained by the author(s) and / or other copyright owners and it is a condition of accessing these publications that users recognise and abide by the legal requirements associated with these rights.

Take down policy

The Research Portal is Queen's institutional repository that provides access to Queen's research output. Every effort has been made to ensure that content in the Research Portal does not infringe any person's rights, or applicable UK laws. If you discover content in the Research Portal that you believe breaches copyright or violates any law, please contact openaccess@qub.ac.uk. 


\title{
DEM modelling of a new 'Sphere Filling' Approach for Optimising Motion Control of Rotational Moulding Processes
}

\author{
Jonathan Adams ${ }^{1}$, Dr. Yan Jin ${ }^{1}$, Dr. David Barnes ${ }^{2}$ and Dr. Joe Butterfield ${ }^{1}$ \\ 1 School of Mechanical and Aerospace Engineering, Queen's University Belfast, UK \\ 2 School of Mathematics and Physics, Queen's University Belfast, UK
}

\begin{abstract}
Rotational moulding is a polymer forming process used to create hollow, stress-free products using both heat and rotation. The basic principles behind the machines which execute the process of rotational moulding have not changed significantly over the last 60 years. A factor restricting the growth of the rotational moulding industry is the limited wall thickness uniformity that can be achieved using the current machines which have limited motion control. Improved flexibility of motion control over the mould is now available and will be investigated with the aim of providing a more efficient process and higher quality products. Using a mathematical 'sphere filling' curve approach, a rotational path can be designed which allows every area of a spherical mould to spend a more uniform time period in contact with the powder pool (Wall thickness uniformity is affected by the powder-wall contact time). This paper proposes a new approach to mould motion control optimisation and provides validation using Discrete Element Method (DEM) simulations. This method has been found to increase the uniformity of powder-wall contact time by up to $19 \%$.
\end{abstract}

\section{Introduction And Background}

Rotational moulding is increasingly competitive with other polymer forming processes, namely injection and blow moulding. However, a comparative limitation of rotational moulding is the uniformity of wall thickness that can be achieved (wall thickness for many product shapes is able to be controlled to within $\pm 20 \%$ [1]). This is largely due to the fact that no pressure is used to force the polymer into cavities. Instead, the polymer lays up layer by layer against an internal mould wall under rotation. Wall thickness uniformity is an important factor, as for many applications, the higher the uniformity, the lower the amount of material that is required to achieve a nominal wall thickness of the part. Therefore, material, weight, energy and processing times can be reduced with better wall thickness uniformity.

A number of factors influence the wall thickness uniformity. These include: powder-wall contact time, mould wall 'recharge' time, powder flowability (how well powder can travel into tight spaces), mould geometry (powder can be held up at corners or protrusions), heating system (uniformity of heat supply to mould), 
mould mount (shielding heat transfer to mould wall), mould wall thickness variations (decreasing the mould surface temperature uniformity), warpage effects, and presence of bubbles [2]. The key factor that controls the wall thickness uniformity is the powder-wall contact time where the areas that see the most of the powder bed are expected to be the thickest.

A method of creating a more uniform wall-powder contact time is to rotate the mould in such a manner that every area of the mould will spend an equal time period in and out of the powder pool. Motion control parameters, which are the rotational speeds about two perpendicular axes for bi-axial rotational machinery, are the key factors that affects the uniform distribution of the powder across the mould surface [3]. The mould rotation is currently limited to two degrees of freedom (2-DOF) with both axes having to rotate at constant speeds throughout the cycle. This restriction of mould rotation and speed is a limiting factor in obtaining optimised uniformity of powder-wall contact time. Crawford et al. [4] predicts that in future advances of motion control, the rotational speed and speed ratios will not be fixed to improve uniformity.

While the rotational speed (ranging from 4-30 RPM [5]) is important to provide the optimum powder flow conditions at the bottom of the mould $[6,7,8]$ and to allow all areas of the mould surface sufficient time to 'recharge', it is the speed ratio between the two perpendicular axes that largely dictates the powderwall contact time uniformity. The speed ratio determines the number of times a specific area on the mould wall passes through the powder bed and the direction in which it enters and exits the powder bed. As well as wall thickness uniformity, Ramkumar et al. $[9,10,11]$ found that choosing the correct speed ratio is crucial for the mechanical properties of the finished part such as tensile, impact and flexural strength.

For a given mould geometry, a rotational speed ratio is chosen to obtain a suitable uniform (or non-uniform) wall thickness $[12,7]$. The thickness of plastic on the mould wall is dependent on how often each point on the mould surface passes through the powder bed and for how long. Due to machinery limitations and a lack of technical understanding, little has been achieved in this area beyond a wasteful trial and error approach to find suitable speed ratios (E.g. 4:1 for a sphere, 8:1 for a horizontally mounted cylinder, and 1:5 for a vertically mounted cylinder) for certain mould geometries using the conventional bi-axial rotational moulding machine.

Conventional bi-axial rotational moulding machines are limited to two degrees of freedom and many can only be used for a constant rotational speed about two perpendicular axes, i.e. they lack the functionality to be controlled to alter rotational speeds effectively throughout a moulding cycle. Robotic technologies have the potential to fundamentally change and significantly improve production practices through the introduction of automated processing and digital control benefits but they need to be proven as being technically and commercially viable for rotational moulding. AMS [13] have developed a robot arm which can hold an electrically heated mould. This lifts the restriction and opens a new potential for improving product quality, namely wall-thickness uniformity. 
Discrete Element Method (DEM) RotoSim is the only commercially available simulation package of rotational moulding processes. However, it lacks a powder flow model and is limited to constant speed bi-axial motion control. The discrete element method (DEM) is a means of simulating the flow of powder and granular matter. This is achieved by modeling a number of discrete particles and calculating the contact and non-contact forces acting on each particle over a period of time. For every particle, its position, velocity and acceleration are found using Newton's equations of motion. Nguyen et al. [14] were the first to use DEM for modeling rotational moulding processes. They used DEM to simulate the flow and heat transfer within the powder bed in a uni-axial rotating cylinder during the initial stage of a rotational moulding cycle (up to point of polymer powder starting to adhere to mould wall). The aim is that the discrete element method can be used to replace current trial and error optimisation techniques with predictive methods which optimise conditions and enable Industry 4.0 practices around automated control and data management.

Knowledge Gap There has been no research to date investigating the possible benefits from utilizing the improved motion control of bi-axial rotation via altering the speed or utilizing increased flexibility of mould rotation beyond 2-DOF. How to utilise this increased flexibility of mould rotation to improve part quality and cycle times is an unknown. Using a mathematical method to utilise the increased flexibility and control of the mould rotation by designing an optimised rotational path is a new and challenging task.

\section{Mathematical Foundation and Hypothesis}

The lack of rotational flexibility and control within current rotational moulding machines, restricts the uniformity of powder-wall contact time that can be achieved. This hypothesis assumes that improving the uniformity of powderwall contact time will lead to enhanced product wall thickness uniformity. With the aim to improve powder-wall contact time uniformity, a new rotational path will be defined. This path will bring all areas of the mould surface through the bottom-most point of the mould (assumed to be the core of the powder bed) in regular equal time periods. It is predicted that this approach will improve on the uniformity currently achieved through conventional speed ratios.

Taking a sphere shaped mould as a case study, if its surface is covered with a number of regular equidistributed nodes (Fig. 1), the problem can be clearly visualized, which is how best to rotate the mould as to bring each of the nodes through the powder pool (at the bottom of the mould) to achieve contact time uniformity.

\subsection{Sphere Filling Curves}

Gerlach and von der Mosel [15] investigated a mathematical approach of how to cover the surface of a sphere with a closed curve and a constant thickness without 


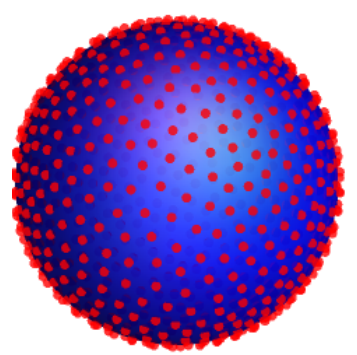

Figure 1: Sphere mould surface covered by equidistributed nodes

overlaps or self-intersection. They found that by plotting a series of latitudinal circles on the sphere surface an equal circumferential distance apart (number of circles depending on the desired 'thickness' of the curve), splitting the sphere into a western and eastern hemisphere, and rotating the western hemisphere by a specific angle, that the longest closed curve of a specified minimum thickness is found which covers the mould surface.

Figure 2 shows the curves covering a sphere for a range of circles. The number of circles, $n$, depends on the minimum thickness of the curve that is desired. The curves are shown in Fig. 2 with the same thin thickness. If the thickness is increased for each curve they would cover the sphere completely before coming into contact with another part of the curve. The number of circles is chosen according to the thickness or width of the powder bed which is expected to provide a uniform covering across the mould surface.

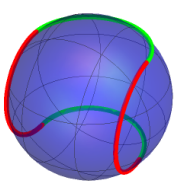

(a) $n=2$

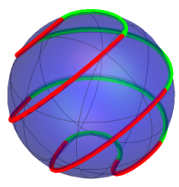

(b) $n=4$

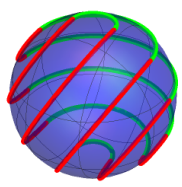

(c) $n=6$

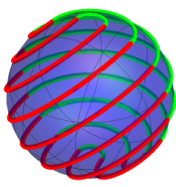

(d) $n=9$

Figure 2: Sphere filling curves with varying circles, $n$

Figure 3 shows the paths that the bottommost point of the sphere mould travels for a range of speed ratios which use constant speeds. Comparing these paths to the sphere filling paths shown in Fig. 2, it can be seen how a more uniform powder-wall coverage can be expected using the sphere filling curve approach.

From analysing these constant speed ratios, it can be expected that it is at the poles of the sphere mould (or rather the areas of the mould surface around 


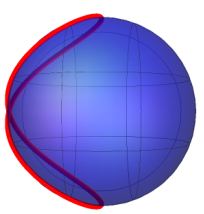

(a) $1: 1$

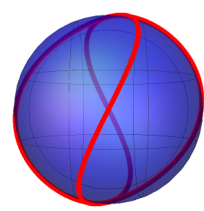

(b) $2: 1$

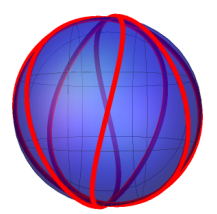

(c) $4: 1$

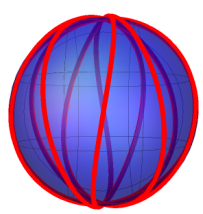

(d) $6: 1$

Figure 3: Trace of lowest point on sphere surface during constant speed biaxial rotation for range of speed ratios

the secondary axis of rotation) that see the most traffic as it is here that the path crosses over and intersecs on itself. This pattern of increased traffic at the poles cannot be changed for any constant speed bi-axial rotation, which limits the uniformity that can be achieved using this approach.

\section{New Motion Control Method for Spherical Mould Rotation}

\subsection{Designing the Rotational path}

Gerlach and von der Mosel describe a curve that covers a sphere's surface (given a minimum curve thickness) as a series of semi-circles. In order to turn these series of semi-circles into one continuous path the order and direction in which each semi-circle follows must be defined. To achieve this, Mathematica was used to map the coordinate at the end of each semicircle to a coordinate at the start of another. Using this approach the correct sequence of semi-circles can be found (Fig. 4(a)).

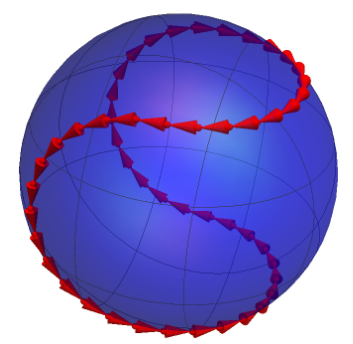

(a) Defining path direction

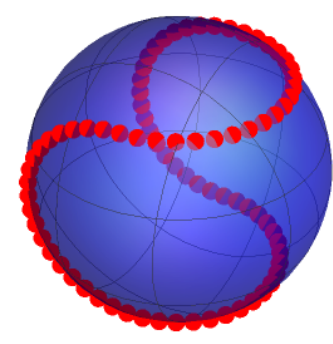

(b) Filling path with nodes

Figure 4: Defining path of a sphere filling curve 
The next step is to rotate the sphere so that the bottom-most point of the sphere (assumed to be core of the powder bed) traces out the sphere filling curve. To achieve this, a series of nodes were plotted along the sphere filling path (Fig. 4(b)). With knowing the co-ordinates and the sequence of the nodes, a pointto-point approach was used to find the spherical co-ordinates to rotate the next node in the sequence to the bottom point of the sphere $([0,0,-1]$ for unit sphere). This was repeated until all the nodes have been passed sequentially through point $[0,0,-1]$ to provide a list of rotations about two perpendicular axes.

\subsection{Development of DEM to Model Rotational Moulding Processes}

Currently, the only simulation package that provides a powder-wall contact uniformity is with RotoSim. RotoSim can be used to calculate the powder-wall contact time uniformity. However, as previously discussed, RotoSim does not have a powder flow models incorporated within it. Rather, it assumes that the powder fills the mould from the bottom most point up to a horizontal surface.

The use of DEM as a new method of obtaining the powder-wall contact uniformity is proposed in this paper. DEM holds two key advantages. Firstly, varying rotation speeds and speed ratios required to rotate the sphere through the sphere filling path can only be modeled with DEM. Secondly, DEM provides a more accurate representation of the position and flow of the powder.

In order to use DEM to find the powder-wall contact time, a way of determining (for every defined time period) whether or not a mould element is in contact with the mould wall must be found. One method of achieving this is to track the position of each element during the rotation and if the position of the element is within a certain distance (particle radius) of any particle then it is said to be in contact at that time. However, this approach was found to be too computationally intensive. Another approach is to utilise a function within the DEM package, LIGGGHTS [16], which is to record the pressure being applied to each mould element in the mesh. A non-zero pressure reading on the mesh element represents contact with powder particles. This method was used as it is less computational. The output files from the DEM simulations were then manipulated to find the powder-wall contact time for each element in the mesh over the simulation.

\section{Simulation Setup}

Discrete Element Method(DEM) was used to investigate and compare the powderwall contact time uniformity of rotating the sphere by the novel rotational paths proposed in Sect. 2.1 and the conventional bi-axial rotations. The DEM package used for these simulations was LIGGGHTS [16]. This paper will focus on investigating the benefit of using the sphere filling curve approach which uses 2 circles, $n$ (Fig. 2(a)). 


\subsection{Procedure}

The procedure to find the powder-wall contact time is detailed below.

1. Construct mould geometry. Model sphere using CAD and create a mesh. Insert mesh into LIGGGHTS environment specifying position and orientation relative to the axes of rotation.

2. Insert powder particles. Specify particle insert region within the mould and allow particles to fall to the bottom of mould under gravity (Powder parameters as per Table 1).

3. Rotate mesh. Once particles have settled begin the rotation of the mesh. Sphere mould rotated using a 4:1 speed ratio and a sphere filling curve rotation $(2 n)$.

4. Record pressure values. Record values of each mesh elements every 1000 time-steps (every 0.01 seconds). Non-zero pressure values represents contact with the powder. This information is used to find powder-wall contact time.

\subsection{Input Variables}

DEM input parameters are shown in Table 1. These parameters were obtained from Nguyen et al. [14] who simulated and analyzed powder flow of HDPE during the initial heating stage of the rotational moulding cycle using DEM. Note that spherical shaped particles were chosen to aid simulation time. In reality, particle shapes are irregular.

Table 1: Parameters used in DEM simulations.

\begin{tabular}{c|c|c} 
& Value & Unit \\
\hline Radius of Mould & 0.05 & $m$ \\
Particle Density & 965 & $\mathrm{Kg}^{-3}$ \\
Particle Radius & $50-350$ & $\mu \mathrm{m}$ \\
Young's Modulus & $1.4 .10^{6}$ & $\mathrm{~Pa}$ \\
Poisson Ratio & 0.45 & \\
Particle-Particle Friction & 0.5 & \\
Particle-Mould Friction & 0.5 & \\
Coefficient of Restitution & 0.4 & \\
Time-Step & $1.10^{-5}$ & $s$ \\
Particle Shape & Spherical & \\
Powder Load & $5-30$ & $\%$
\end{tabular}

For the sphere mould, the literature suggests that a speed ratio of 4:1 is optimum. Therefore, the results from the 'sphere filling' rotations will be compared to the $4: 1$ speed ratio. A range of powder loads from $5 \%$ to $30 \%$ will be tested, which is common within rotational moulding. 


\section{Results}

Table 2 shows the average element time over the mould for a $4: 1$ speed ratio rotation and a 2 circle sphere filling curve with a $25 \%$ powder fill. At this fill level, the sphere filling approach is found to reduce the standard deviation of contact time when compared to a 4:1 speed ratio. For 4:1 speed ratio, it can be seen how the areas at the poles spend most time in contact with the powder bed compared to the areas around the equator. This was expected and be correlated to the path of the lowest point (See Fig. 3), where the path insects itself at the poles. For the sphere filling approach, the opposite trend was observed, where the areas around the poles were seen to spend the least time in contact with the powder bed.

Table 2: Comparison of DEM Outputs for Sphere Filling Curve and 4:1 Speed Ratio Rotations

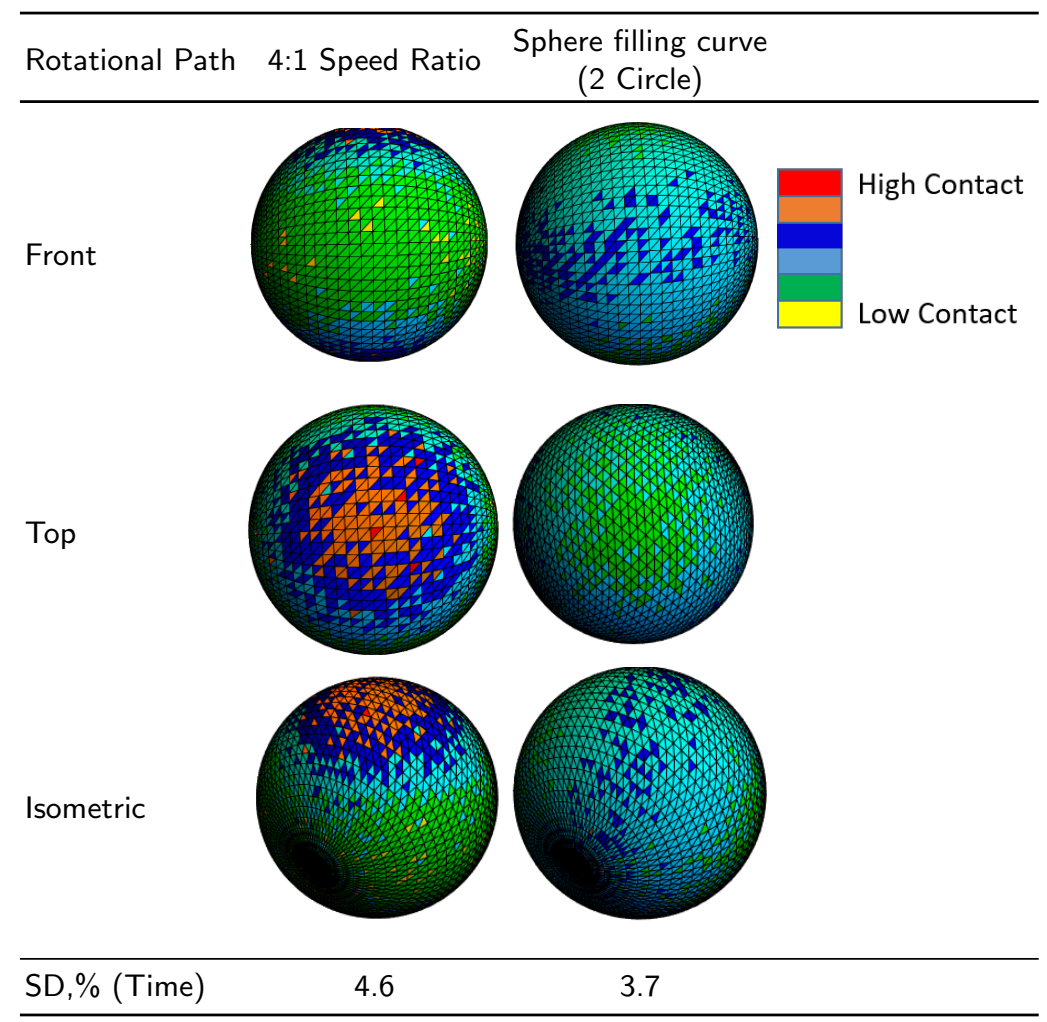

Results (Fig. 5) show that for powder fills between 20-30\% using the sphere filling approach increases the uniformity of powder-wall contact between the powder bed and the mould wall (compared to the conventional constant speed 


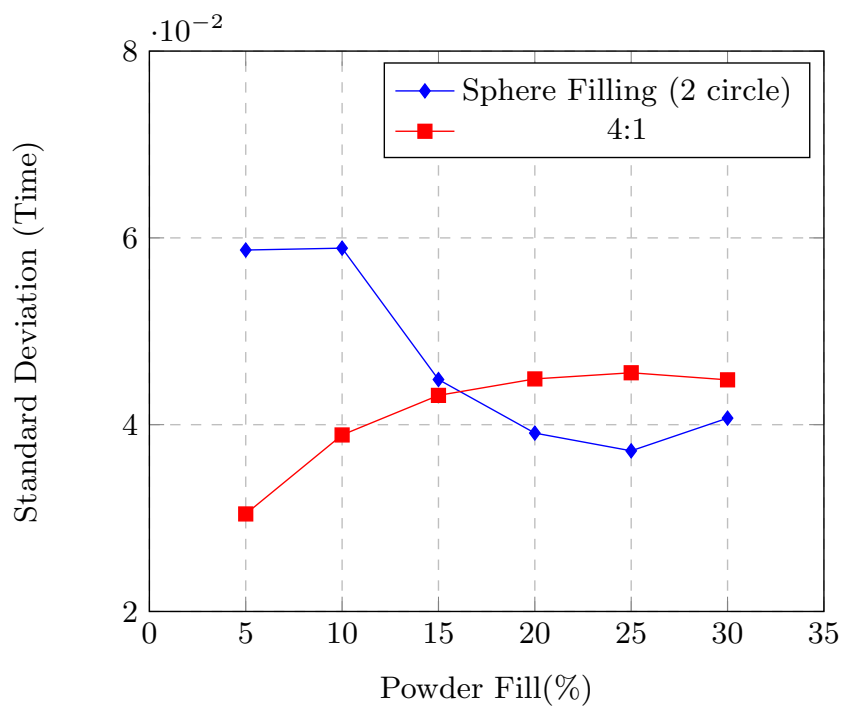

Figure 5: Contact Time Uniformity from sphere filling and 4:1 speed ratio rotations for range of powder fills.

4:1 speed ratio). As the powder fill level drops below 20\%, the conventional 4:1 ratio was found to have better contact time uniformity (Fig. 5). This finding could be due to the width of the powder bed dropping below the minimal width needed to cover the mould surface with 2 circle, $n$. It is predicted that increasing the circle number with decreasing powder fills will improve uniformity.

\section{Conclusions And Future Work}

This research is the first to investigate a mathematical 'sphere filling' approach to define a path in which to rotate a mould during the rotational moulding heating cycle. A numerical model of powder flow (DEM) was used to demonstrate the advantages of this mathematical approach by studying the powder-wall contact uniformity, which affects the wall thickness uniformity. It was found that with the 'sphere filling' method (for the case of 2 circles, $n$ ), it can increase the powderwall contact uniformity for a spherical mould by up to $19 \%$, when compared to the conventional 4:1 speed ratio. For powder fill levels below 20\%, 4:1 speed ratio was found to be better. It is predicted that increasing the number of circles (Fig.2) will improve the powder-wall conact uniformity at these powder levels.

These findings open a new avenue of research into altering the rotational speed ratio to achieve a designed rotational path throughout the heating cycle to optimise the uniformity of wall thickness. Future work will investigate increased number of circles in the 'sphere filling' curves and further validate the benefits of the 'sphere filling' method with experimental testing. This space-filling approach will be developed to other mould geometries beyond a sphere. 


\section{Acknowledgments}

The authors thank the Department of Education and Learning (DEL) for funding provided for this research project.

\section{References}

1. Crawford.R. J., Rotational moulding of plastics. Research Studies Press Ltd., 2 ed., 1996.

2. K. Walls, The dimensional control of rotationally moulded products. PhD thesis, Queen's University, Belfast, 1998.

3. A. Roa and J. Throne, "Principles of Rotational Molding," Polymer Engineering E Science, vol. 12, no. 4, pp. 237-264, 2004.

4. R. J. Crawford and M. Kearns, "The Future for Rotational Moulding," in Practical Guide to Rotational Moulding, ch. 6, pp. 163-169, Smithers Rapra Press, 2003.

5. R. J. Crawford and S. Gibson, "Rotational Molding: A Review," Popular Plastics E Packaging, pp. 19-23, 2015.

6. J. Olinek, C. Anand, and C. T. Bellehumeur, "Experimental study on the flow and deposition of powder particles in rotational molding," Polymer Engineering and Science, vol. 45, no. 1, pp. 62-73, 2005.

7. A. A. Aissa, C. Duchesne, and D. Rodrigue, "Characterization of Polymer Powder Motion in a Spherical Mold in Biaxial Rotation," Polymer Engineering Es Science, vol. 52, no. 5, pp. 953-963, 2012.

8. L. Ma, C. Wang, and W. Yang, "Heat Transfer during Rotational Molding of a Single Axis Device," Advanced Powder Technology, vol. 87, pp. 116-118, 2010.

9. P. L. Ramkumar, D. M. Kulkarni, and V. V. Chaudhari, "Parametric and mechanical characterization of linear low density polyethylene (LLDPE) using rotational moulding technology," Sadhana-Academy Proceedings in Engineering Sciences, vol. 39, no. 3, pp. 625-635, 2014.

10. P. L. Ramkumar, A. Ramesh, P. P. Alvenkar, and N. Patel, "Prediction of heating cycle time in Rotational Moulding," Materials Today: Proceedings, vol. 2, no. 4-5, pp. 3212-3219, 2015.

11. P. L. Ramkumar, S. D. Waigaonkar, and D. M. Kulkarni, "Effect of oven residence time on mechanical properties in rotomoulding of LLDPE," Sadhana - Academy Proceedings in Engineering Sciences, vol. 41, no. 5, pp. 571-582, 2016.

12. R. A. Pethrick and N. E. Hudson, "Rotational moulding a simplified theory," Proceedings of the Institution of Mechanical Engineers, Part L: Journal of Materials Design and Applications, vol. 222, no. 3, pp. 151-158, 2008.

13. M. Trebing, "AMS Robotics - Robomould." https://www.rotomolding.org/pdf/Fully.pdf, 2017.

14. H. T. Nguyen, B. Cosson, M. F. Lacrampe, and P. Krawczak, "Numerical simulation on the flow and heat transfer of polymer powder in rotational molding," International Journal of Material Forming, pp. 423-438, 2014.

15. H. Gerlach and H. von der Mosel, "On sphere-filling ropes," The American Mathematical Monthly, pp. 1-15, 2010.

16. C. Kloss, C. Goniva, A. Hager, S. Amberger, and S. Pirker, "Models, algorithms and validation for opensource DEM and CFD-DEM," Progress in Computational Fluid Dynamics, vol. 12, no. 3, p. 140, 2012. 